\title{
PERCEPCIÓN DE ENFERMERÍA RESPECTO A LA ATENCIÓN HOSPITALARIA OTORGADA A PERSONAS QUE VIVEN CON VIH
}

NURSING PERCEPTION REGARDING THE ATTENTION GIVEN IN HOSPITALS TO PATIENTS THAT LIVE WITH HIV

PERCEPÇÃO DA ENFERMAGEM EM RELAÇÃO A ATENÇÃO HOSPITALAR DADA PARA PESSOAS QUE VIVEM COM HIV

Silvana del Carmen Castillo Parra

Universidad de Chile. Chile

scastill@med.uchile.cl

ORCID: 0000-0002-9195-1391

Denisse Carolina Quijada Sánchez

Universidad de Chile. Chile

ORCID: 0000-0002-4719-2738

Daniela Soledad Feliú Pérez

Universidad de Chile. Chile

ORCID: 0000-0002-3232-5630

Romina Paz Fernández Torres

Universidad de Chile. Chile

ORCID: 0000-0002-1354-7684

Oriana José Molina Sevilla

Universidad de Chile. Chile

ORCID: 0000-0002-7150-4957

María Fernanda Monsalves Morales

Universidad de Chile. Chile

ORCID: 0000-0003-0430-4813

Victoria Elizabeth Otay Ríos

Universidad de Chile. Chile

ORCID: 0000-0002-1367-0914

DOI: https://doi.org/10.22235/ech.v7i2.1643

Recibido: 18/12/2017

Aceptado: 05/04/2018

Resumen: Dado el contexto epidemiológico en Chile referente al alza de la cantidad de personas con VIH, cobra relevancia el fortalecimiento del cuidado otorgado a estos pacientes. Por esto, el objetivo de esta investigación es conocer la percepción de 
enfermeras(os) respecto a la atención otorgada a personas que viven con VIH (PVVIH) en un servicio hospitalario de atención terciaria de salud, buscando contribuir a la enfermería. Para ello se utilizó un paradigma de investigación cualitativo tipo estudio de caso, recolectando la información con una entrevista semiestructurada, finalizando con un análisis del discurso de tipo semiótico. Se destacó que en las primeras atenciones se generaba "temor" en la atención a PVVIH, el que desaparecía a medida que aumentaban los conocimientos de la profesional. Enfermeras participantes refirieron la inexistencia de diferencias en la atención entre PVVIH y otros pacientes, evidenciándose una incongruencia entre el discurso y el actuar en enfermería al ocupar mayores medidas de precaución al realizar procedimientos a PVVIH. Como fortalezas identificadas en la atención a PVVIH se encontró el no discriminar a pacientes, la capacidad de enfrentar distintos contextos de atención y la destreza procedimental, entre otras, mientras que los aspectos a mejorar en la atención se vincularon con la relación que se genera con los PVVIH y sus familiares.Como conclusión, las enfermeras no hacen mayores distinciones en la atención con PVVIH. Es importante que las profesionales reconozcan sus habilidades y aspectos a mejorar para seguir humanizando la atención a PVVIH, siendo agentes educadores y de cambio.

Palabras clave: VIH, Enfermería, Enfermeros, Percepción, Relaciones EnfermeroPaciente, Atención a la Salud.

Abstract: Given the epidemiological context in Chile concerning the increased quantities of people with HIV, improving the care given to these patients becomes relevant.

The objective of this research is to find about the perception of nurses concerning the care given to people living with HIV (PLHIV) in a tertiary health care hospital service, seeking to contribute to nursing improvements. In order to accomplish this task a qualitative research paradigm was used as a type of case study, collecting the information with a semistructured interview, ending with a semiotic analysis of the discourse. It was pointed out that in the first sessions there is some fear generated in the attention to PLHIV, which gradually disappears with the increase of the professional's knowledge. Participating nurses referred to the lack of differences in care between PLHIV and other patients, evidencing an incongruence between the speech and the action by taking greater precautions when performing procedures for PLHIV. The strengths identified in the attention to PLHIV were the no discrimination to these patients, the ability to face different contexts of attention and the procedural skills, among others, while aspects to improvement in care were linked to the relationship that is generated with PLHIV and their families. In conclusion, nurses do not make noticeable distinctions in the care of PLVIH. It is important that professionals recognize their skills and the aspects that can be improved in order to continue humanizing the attention to PLHIV, acting as educators and agents of change.

Key words: HIV, Nursing, Nurses, Perception, Nurse-Patient Relations, Health Care.

Resumo: Dado o contexto epidemiológico no Chile referente ao aumento das pessoas com HIV, é relevante a necessidade de fortalecer os cuidados desses pacientes. Devido a isso, o objetivo de esta pesquisa é conhecer a percepção de enfermeiros(as) em relação a atenção 
fornecida para as pessoas que vivem com HIV num serviço hospitalar de atenção terciária em saúde, buscando contribuir para a disciplina e profissão de enfermagem. Para isso se utilizou um paradigma de pesquisa qualitativo tipo estudo de caso, o meio para reunir informação foi a entrevista semiestruturada, finalizando com uma análise de discurso. Destacou que nas primeiras atenções de pessoas portadoras de HIV o "temor" era perceptível. "Temor" que desaparecia à medida que o conhecimento do profissional aumentava. Enfermeiros participantes falaram que não existe diferença alguma na forma de atenção de pacientes portadores de HIV, mas se notou uma incongruência entre o discurso e a atuação da enfermagem ao tomar maiores medidas de precaução no momento de realizar procedimentos com esses pacientes. Pontos fortes identificados na atenção de portadores de HIV foram a não discriminação dos pacientes, a capacidade de enfrentar-se a distintos contextos na atenção, as habilidades de procedimentos, entre outras. Enquanto aos aspectos a melhorar na atenção se vincularam com a relação que se gera como os pacientes portadores de HIV e seus familiares Como conclusão os enfermeiros( não fazem maiores distinções na atenção de pacientes com HIV. É importante que os profissionais reconheçam suas habilidades e pontos para melhorar e assim continuar avançando na humanização da atenção dos portadores de HIV conseguindo ser agentes educadores e de mudança.

Palavras-chave: HIV, Enfermagem, Enfermeiros, Percepção, Relações Enfermeiro Paciente, Atenção a Saúde.

\section{INTRODUCCIÓN}

Las cifras internacionales entregadas por ONUSIDA revelan que en 2014, 36,9 millones de personas en el mundo vivían con el Virus de Inmunodeficiencia Humana (VIH), mientras que en América Latina las nuevas infecciones alcanzaron cifras de aproximadamente 87.000 casos, descendiendo un $17 \%$ con respecto al año 2000 (1). En cuanto a Chile, se ha evidenciado un aumento progresivo de los casos notificados de $\mathrm{VIH}$, llegando a un 44,6\% de aumento en 2015 respecto al año 2010, donde una cifra importante se encuentra en etapa SIDA al momento de la confirmación (2). En consecuencia, muchos deben ser tratados en el servicio de atención terciaria, donde el profesional de enfermería adquiere un rol fundamental en el cuidado del paciente. Según Vidal, Adamuz y Feliu el establecimiento de la relación terapéutica es clave a la hora de brindar cuidados; por tal motivo el cuidado puede verse afectado según la percepción que la profesional tenga sobre la persona, y por consiguiente, puede alterar el proceso de salud-enfermedad de la persona que vive con VIH (PVVIH) $(3,4)$.

Se buscó analizar las experiencias, identificar las herramientas y conocer las fortalezas y aspectos a mejorar propios de las profesionales de enfermería en la atención de PVVIH para así lograr el objetivo de conocer la percepción de éstos respecto de la atención otorgada a estas personas en los servicios de atención terciaria de salud en Chile. Con lo anterior se pretende contribuir a la enfermería a través del fomento de la reflexión que debe realizar el profesional en su quehacer para brindar cuidados humanizados a la PVVIH. 


\section{METODOLOGÍA}

Se realizó una investigación cualitativa de tipo estudio de caso, la cual permite abordar de forma más profunda y en su contexto una determinada situación, permitiendo así su mayor comprensión (5). Esta investigación se llevó a cabo en un hospital de la región Metropolitana de Santiago de Chile, entre marzo y noviembre del año 2017. La elección de las(os) participantes fue a través del muestreo por conveniencia, dependiendo básicamente de la accesibilidad a las(os) participantes (6). Junto con esto se utilizó el principio de saturación de datos, donde se recolectó información hasta encontrar argumentos repetitivos en el discurso y que conllevaron al cumplimiento de los objetivos. Se contó con siete participantes enfermeras(os) entre 25 y 45 años, todas(os) pertenecientes a los servicios de medicina del hospital y con un promedio de cuatro años en la institución. Como criterios de inclusión se consideró que las(os) enfermeras(os) hayan atendido a PVVIH, tuvieran como mínimo dos años de egresadas(os) de la profesión y un año de antigüedad mínimo dentro del hospital. Se consideró como exclusión enfermeras(os) que hubiesen presentado licencia médica durante el proceso de selección y/o desarrollo de la investigación.

Para esta investigación se contemplaron los requisitos básicos de ética de la investigación planteados por Ezekiel Emanuel, los cuales se expusieron en el consentimiento informado, especificando el resguardo de la identidad e información entregada (7). Éste fue leído y explicado previo a cada entrevista, siendo firmado por las(os) participantes, otorgando así la autorización de participación. Los criterios de rigor que se consideraron para el desarrollo de esta investigación fueron los expuestos por Egon G. Guba (8). La credibilidad o valor de la verdad se contempló al interpretar lo más fielmente posible el discurso otorgado por las(s) participantes, triangulando la información por investigadoras que no participaron en el desarrollo de la entrevista. En cuanto a la transferibilidad o aplicabilidad, se describió el contexto hospitalario y de las(os) participantes y se espera que la información obtenida de esta investigación pueda ser útil en otros contextos o sirva como base para futuros estudios contribuyendo a la enfermería. Por otro lado, la confirmabilidad o neutralidad se respetó realizando transcripciones exactas de las entrevistas de las(os) participantes y utilizando citas textuales en el análisis de la información. Finalmente, la dependencia o consistencia se abarcó desde las investigadoras al momento de las entrevistas estableciendo una escucha activa frente al relato de las(os) participantes, omitiendo juicios de valor o frases que pudiesen inducir una respuesta determinada por parte de las(os) mismos.

El método de recolección de información se realizó por medio de la elaboración y aplicación de una entrevista semiestructurada, la cual presenta mayor grado de flexibilidad ajustándose a cada participante (9). Cada entrevista se llevó a cabo por dos investigadoras en lugares dentro del hospital donde se resguardó un ambiente íntimo. La duración de éstas fue en promedio de 30 minutos.

La metodología empleada para el análisis de la información fue el análisis del discurso (10) de tipo semiótico, donde las investigadoras transcribieron las entrevistas, que luego fueron codificadas. A través de pasajes textuales se inició el estudio de éstas con el fin de clasificar 
el discurso en categorías y subcategorías emergentes. Posteriormente se elaboró una tabla de clasificación donde se asoció toda la información relevante tras el análisis realizado, lo cual se utilizó para extraer un análisis final relacionando todas las entrevistas y estableciendo así las conclusiones finales.

Se recalca que esta investigación fue aprobada por el Comité de Ética para Investigación en Seres Humanos, con Acta de Aprobación del proyecto No 105-2017.

\section{RESULTADOS}

Los resultados de esta investigación se dividieron en seis categorías (herramientas, percepción, primeras vivencias, relación enfermera/o - paciente, fortalezas, aspectos a mejorar) y subcategorías que describieron más minuciosamente las categorías anteriormente señaladas; posteriormente toda esta información se relacionó entre sí para exponer los resultados de una forma más integra. A continuación se presentan los resultados donde se exponen citas textuales de las entrevistas realizadas, las que se encontrarán en cursiva. Al inicio, estas citas presentarán un código que incluye el número de la entrevista y la ubicación de cada cita en dicha entrevista.

Analizando el discurso de las(os) participantes, en cuanto a la formación de pregrado refirieron haber recibido educación con un enfoque holístico, con visualización integral de la persona y su bienestar biopsicosocial, donde el mayor énfasis se centraba en los contenidos científicos desde las ciencias biomédicas hasta lo relacionado con la disciplina de enfermería. En cuanto al VIH, se destacó un enfoque relacionado principalmente al manejo de la patología.

E2. (76-80): "Los PAE (...) harta anatomía, fisiopato en realidad, y a raíz de eso uno toma los conocimientos que tiene y los respalda básicamente. Eso fue lo que más se nos recalcó a nosotros."

Continuando con la formación ética, se mencionó tanto la existencia de ramos específicos a esta temática como también su abordaje transversal, considerándola parte esencial en el cuidado del paciente y recalcando la importancia de la confidencialidad como parte de ella.

E1. (191-193): “(...) lo más importante siempre va a ser la ética. Uno tiene que saber que el diagnóstico del paciente es privado totalmente; si él no quiere que la familia lo sepa, entonces la familia no tiene porqué enterarse."

Conjuntamente, se dio énfasis al desarrollo de habilidades en torno al cuidado humanizado como la empatía, trato amable, establecimiento de una relación terapéutica y mantener al paciente informado, destacando en cuanto a la percepción del cuidado, que éste debiese ser siempre igual para todos, sin diferencias al trato otorgado a las PVVIH. Resaltó la importancia del refuerzo de estas habilidades desde el pregrado, para así poder entablar una relación terapéutica con todos los pacientes.

E7. (121-126): "Porque eso se trabaja del cuidado, porque cuidar no solamente es poner un apósito, cuidar es también estar pendiente de la sensación del paciente, si 
está triste, aburrido, eufórico, o sea, sino eso hace parte del cuidado. Así que eso está relacionado. Porque uno está tratando con persona, no tratando con una hoja, que no siente nada, que da lo mismo si la arrugas; no, uno tiene que tener harto de tacto con la gente, porque no, asi eso siempre, siempre, siempre."

Sin embargo, se apreció una diferenciación en la atención hacia PVVIH, en cuanto al uso de las medidas de precaución estándar, existiendo una postura que hacía énfasis en la necesidad mayor de protección al realizar procedimientos a PVVIH y otra, que manifestó que estas medidas son necesarias en procedimientos a cualquier paciente.

E2. (178-180): "Por ejemplo, no es lo mismo hacer un procedimiento a un paciente que es positivo, o con algún tipo de virología positiva, a un paciente que no lo tiene, ¿ya? Uno lo hace con más cuidado, con mayor precaución."

E5. (150-155) "En realidad es lo que te comentaba, es un paciente más, nomás. No hago la diferencia como que "el paciente que vive con VIH". Sí, claro, obviamente uno tiene como más precaución. De repente, uno por costumbre, tiene la pinta de pinchar a un paciente sin guantes. De hecho, ahora último igual como que tomamos un poco más de conciencia, en general; por ejemplo ahora yo no punciono a un paciente sin guantes, sabes que aunque me haya tocado VIH o no sea VIH, no me arriesgo a... o sea, me protejo ante un eventual accidente cortopunzante o punción con alguna bacteria, o algo (...)"

Al cuidado se suma la relación enfermera/o - paciente donde se mencionó la dedicación y paciencia que se debe tener en cada atención, junto con lo dificultoso que es el no encariñarse con los pacientes, evidenciándose que muchas veces esta relación se ve afectada por los pacientes, quienes en ocasiones son más reacios a la atención.

E5. (211-214) “(...) igual uno es como inevitable de repente encariñarse, de repente no sentir nada, o al contrario, de repente vamos a encontrar de todo como que .... Si, hay pacientes que tienden a ser un poquito más hostil, pero igual uno los va a atender de la misma manera ... va un poco en el tema de las relaciones personales."

Refiriéndose a las PVVIH se destacaron las complicaciones en su tratamiento debido a lo que implica la misma patología y la falta de adherencia farmacológica percibida por las(os) profesionales de enfermería. Sin embargo, se considera que las PVVIH pueden lograr una buena calidad de vida si asisten a sus controles de salud y tienen su tratamiento farmacológico al día.

E7. (245-251): "El tema es que hay poca adherencia al tratamiento, hay gente que no le ve la importancia de tomarse un tratamiento antirretroviral, no ve la importancia de continuar el tratamiento, de ser como riguroso, de tomárselo todos los días. Yo creo que hay también de pronto un desconocimiento de las reacciones adversas que tienen lo retrovirales; entonces la gente, "ah no, es que me duele la 
guata, diarrea, ah, entonces me lo dejo de tomar", entonces como que no saben de que esos son efectos secundarios de tomar su terapia."

En torno a los primeros acercamientos a una PVVIH, las(os) participantes los describieron como complejos, coincidiendo en las precauciones en el acercamiento y realización de procedimientos, siendo más meticulosas(os) en su actuar. Destacó la palabra "temor", que luego con el tiempo iba desapareciendo, ya que entendían que la PVVIH no debía ser tratada diferente. Por lo mismo plantearon la necesidad de profundizar más sobre el VIH en el pregrado, evitando así el generar miedo en la atención.

E5. (168-171) “(...) no recuerdo así como el momento exacto, pero sí yo recuerdo que como por el prejuicio que igual uno siente, como el temor de contagiarse, por lo que conlleva la enfermedad en sí y todo, sí recuerdo como más temor, o sea no sé si temor, como estaba como más ojo, como más precaución, qué se yo (...)"

Con respecto a la visión de las(os) participantes sobre la evolución del VIH, se consensuó un cambio a través de los años, destacando que hace un tiempo atrás el VIH era mortal, por lo que las medidas de precaución tomadas eran mucho mayores, siendo incluso algunas innecesarias considerando el mecanismo de transmisión del virus. Esto va en contraste con la realidad actual, donde es más considerada como una enfermedad crónica. Se relacionó el aumento de los casos con la pérdida del miedo de la población a esta infección, y además por el déficit de educación sexual a nivel poblacional tanto desde la prevención a las personas en general, como a las PVVIH para evitar la propagación del virus y fomentar su autocuidado.

E4. (126-131): “(...) uno vivió la etapa del VIH cuando se quemaban colchones porque los pacientes morían, había que meterlos adentro de unas bolsas especiales... era todo un show, pero tú ya vives, uno vive toda esa evolución para delante, entonces ahora el VIH es como... tú ya sabes que no es mortal, que es como una enfermedad crónica, entonces ya no, pero cuando tú llegas a trabajar ya como enfermera se te ha quitado todo el miedo para atrás, ya no tienes ese miedo que tienen ustedes recién egresados."

Al consultar por las fortalezas que las(os) participantes tenían, concordaron que la principal era el poder enfrentarse a diferentes escenarios y pacientes, el poder decir las cosas y llegar a las personas al momento de entregar cuidados. Junto a esto, el hecho de no discriminar a los pacientes, ser siempre cercanos en el cuidado e ir adquiriendo con los años nuevas destrezas procedimentales.

E5. (225-227) “(...) uno va desarrollando más el tacto, más habilidades blandas, ehh... uno ya empieza a ver cómo llegar a él, de repente bromear con los pacientes, y ahí como que uno va tanteando el terreno... y claro, con respecto como a los procedimientos uno va aprendiendo más (...)" 
En cuanto a los aspectos por mejorar, las(os) participantes destacaron en ellas(os) mismas(os) la actitud frente a los familiares y pacientes, haciendo énfasis en las distintas personalidades que se tienen que enfrentar. Se destacó el hecho de que siempre es posible mejorar.

E1. (141-143): "yo creo que siempre hay cosas por mejorar. Pero ya va más que nada en el hecho de que a veces uno se cansa de la actitud de la persona, o se aburre un poco de los familiares y esas cosas, hay que mejorar un poco la actitud frente a eso."

Se hizo énfasis en la dificultad de la participación del profesional de enfermería en la atención de las personas en general y las PVVIH, fundamentalmente en la falta de tiempo dentro de la jornada laboral para hacerse más partícipe en su proceso de salud-enfermedad.

E3. (187-190): “(...) porque muchas colegas que yo he visto que, colegas que pasan cierto, haciendo lo que hay que hacer y poco contacto más allá tienen, pero no sólo con el paciente que tiene VIH sino que con el paciente en general, a veces somos pocas enfermeras, demasiados pacientes, el tiempo se te hace poco (...)"

Finalmente, las(os) participantes concuerdan en que pese a haber tenido una buena formación académica, todos sintieron miedo al egreso del pregrado; esto relacionado con la inseguridad en sus conocimientos. Por otro lado surgió la necesidad de preparar en el pregrado a las y los futuros profesionales de enfermería para educar a la población con y sin VIH, reforzando así la prevención de la enfermedad y evitar, en parte, la transmisión de ésta.

\section{DISCUSIÓN}

Actualmente en Chile, como indica el Instituto de Salud Pública (ISP), la prevalencia de infectados por VIH va en constante aumento (2). Esta alza, según un estudio realizado por el Ministerio de Salud de Chile (MINSAL), se asoció a conductas sexuales inseguras, una mayor cantidad de parejas sexuales y la poca utilización del preservativo a la hora de tener relaciones sexuales (11). Lo anterior concuerda con lo referido por las(os) participantes, quienes atribuyeron el aumento de los casos de VIH a las conductas sexuales de riesgo, agregando la despreocupación al momento de la prevención del contagio. Además lo atribuyeron al cambio de percepción a través del tiempo que ha tenido el VIH, pasando de ser una enfermedad mortal a ser catalogada en la actualidad como una patología crónica. Asimismo enfatizaron la importancia de la educación sexual en torno a la prevención del contagio, junto con la necesidad de un cambio en el enfoque de la educación a las PVVIH en cuanto a su responsabilidad en el contagio, teniendo conductas sexuales seguras y fomentando su autocuidado.

Es por lo antes mencionado que toma relevancia el cómo se brinda la atención de salud a las PVVIH, por el impacto que ésta puede generar en el control de la enfermedad, la calidad de vida y la prevención de nuevos casos. Bajo este escenario, según un estudio realizado en Cali, Colombia, llamado "Evaluación de conocimientos, actitudes, susceptibilidad y 
autoeficacia frente al VIH/SIDA en profesionales de la salud", se evidenció que el mejorar los conocimientos y habilidades del personal de salud favorece la capacidad para prestar cuidados a las PVVIH (12). Esto concuerda con la presente investigación, donde las(os) participantes coincidieron en que poseer un elevado nivel de conocimiento respecto al VIH les permite brindar una atención de calidad, igualitaria y sin discriminación a las PVVIH.

Asimismo, en el discurso de las(os) participantes se dio énfasis en que el trato hacia las personas debe ser de forma igualitaria, sin hacer distinción según el diagnóstico del paciente. Aún así, éstos explicaron que al efectuar procedimientos a las PVVIH toman mayores precauciones, en comparación a las efectuadas en las personas que presentan serología negativa. De esta forma se evidenció una discrepancia entre el discurso y el actuar de las(os) participantes en donde, por más que se expresó el sentido de igualdad, inconscientemente hicieron diferencias, que en cierta forma podría afectar la manera en la que las PVVIH perciben la atención otorgada.

Por otro lado, en el estudio antes mencionado además se evidenció que las y los profesionales de la salud presentan un cierto grado de temor al momento de atender a PVVIH; esto, pese a tener los conocimientos con respecto a la infección, manteniendo ciertos prejuicios que no les permite involucrarse libremente con ellas. Lo descrito se contrasta con la presente investigación, en donde mediante el análisis desarrollado se dio cuenta que las(os) participantes identificaron esta sensación de "temor" sólo en los primeros encuentros con una PVVIH, ya que el ir interactuando y adquiriendo conocimientos con respecto a la patología les entregó seguridad en su quehacer diario.

En cuanto al desempeño profesional de enfermería, el artículo de revisión "El desempeño de los profesionales de Enfermería" realizado en Cuba y publicado en el año 2016 señala que esto es un ámbito poco abordado en la actualidad, pese a los retos a los que está sometido el profesional de enfermería y las particularidades que existen en el sistema de salud, por lo que se percibe la necesidad de un abordaje profundo del desempeño profesional para así favorecer la gestión del cuidado (13). Esto podría contribuir y enriquecer la enfermería desde el punto de vista de la identificación de las fortalezas y aspectos a mejorar que posee el profesional para otorgar una mejor atención a la persona y lograr una relación terapéutica que contribuya a la salud de éstas.

En otro ámbito, un estudio realizado en Madrid, España en 2016, detalla la importancia de atiborrar el encuentro profesional de significados, por medio de la escucha activa y la validación del paciente (14). Por lo mismo, se menciona que las características del profesional de la salud toman un rol fundamental en este acercamiento, aceptando, ofreciendo respuestas y guiando hacia una solución del problema del paciente, conduciendo a la generación de una relación de confianza y libertad de expresión, ya que el objetivo de la atención de salud no es sólo dar un tratamiento, sino además conseguir "cambiar el miedo por tranquilidad, la vergüenza en normalidad, la confusión en claridad, las dudas en respuesta, el enfado en empatía y la culpa en comprensión” (14). De la misma investigación se identificaron fortalezas por parte del profesional de la salud en la atención de personas con ITS y PVVIH tales como empatía, aceptación de la persona independiente 
de sus pensamientos, escucha activa, no realizar juicios, hablar de sexualidad con normalidad y empoderar a la persona entre otras; esto, en contraposición con las dificultades encontradas en el mismo estudio como el impedimento al hablar de sexualidad, falta de tiempo, juzgar, infantilizar, prejuicios y falta de competencias en comunicación.

Comparando los resultados anteriormente descritos con la presente investigación realizada, las(os) profesionales al consultarles sobre las fortalezas que poseían al atender a las PVVIH coincidieron en que lo principal era su capacidad para enfrentarse a diferentes contextos y personas, el poder expresar lo que piensan y llegar a las personas en el momento de la atención; el no discriminar, ser cercanos y la adquisición de destrezas procedimentales a lo largo del tiempo. Como aspectos a mejorar destacaron la actitud con los familiares y el paciente, haciendo hincapié en las diferentes personalidades que deben ser capaces de enfrentar; además recalcaron que siempre era posible mejorar los cuidados brindados al paciente. En el discurso también surgió la falta de tiempo como factor obstaculizador para que el profesional de enfermería participara activamente en la atención de todas las personas. Este aspecto en relación a la falta de tiempo también fue destacado por la investigación realizada en Madrid, España, mencionando que la presión asistencial continúa siendo una carga insuperable, que imposibilita otorgar el tiempo que muchas situaciones requieren (14).

En base a lo mencionado anteriormente se identificó la existencia de futuros retos para el profesional de enfermería, lo que se alude también en la publicación "Enfermería en el rol de gestora de los cuidados" donde se menciona el desafío que debe asumir la enfermería en cuanto al liderazgo en la gestión del cuidado y la importancia de las habilidades de comunicación y toma de decisiones, siendo esto un punto clave en la formación de futuros profesionales (15). Junto a esto, como menciona Ceballos, es fundamental que desde el pregrado se enseñe a cuidar con empatía y sin importar el diagnóstico o las condiciones del paciente, enfatizando también la importancia del cuidado humanizado el cual permite el crecimiento personal, profesional y dentro del equipo de salud (16).

Finalmente, si bien se identificaron aspectos en el profesional de enfermería que concuerdan con otras investigaciones, tanto nacionales como internacionales, existieron bastantes diferencias en determinados puntos, los que son fundamentales a la hora de atender a una PVVIH.

\section{CONCLUSIONES}

De esta investigación se concluye que las(os) profesionales de enfermería perciben la atención que se les otorga a las PVVIH como igualitaria frente a las personas que presentan serología negativa; sin embargo reconocieron diferencias en cuanto a lo procedimental, enfatizando un mayor cuidado al efectuar procedimientos a PVVIH. Se destaca que sólo se identificó el sentimiento de "temor" en los primeros encuentros con una PVVIH, lo que se contrapone con otros estudios revisados en donde se describe que los profesionales de la salud identificaban el "temor" en el trato con la PVVIH indistintamente de la cantidad de encuentros vividos. Esto sin duda es un avance en cuanto a la atención, ya que se demuestra 
que el conocimiento adquirido por las(os) profesionales de enfermería fundamenta su actuar en la atención y cada día se va alcanzando la igualdad plena en el trato a todas las personas. Además las(os) participantes del presente estudio identificaron como herramientas frente a la atención de las PVVIH, la empatía, trato amable, establecimiento de una relación terapéutica y mantener al paciente informado, lo que fortalece su actuar en el quehacer de enfermería. Todo esto refuerza lo anteriormente mencionado, siendo todas estas herramientas fundamentales en el crecimiento del trato humanizado y en la mejora en la calidad de la atención.

Por otra parte las(os) profesionales de enfermería coincidieron en la importancia de mejorar la actitud hacia la familia y el paciente cuando la relación se torna compleja, siendo esto fundamental para la creación de un lazo de confianza y seguridad mutua en la atención. Junto a esto, refirieron que la falta de tiempo y el sistema de salud en el cual están inmersos les obstaculiza en determinadas ocasiones efectuar un cuidado más acabado a la PVVIH y en general a todas las personas.

Se destaca que mediante el análisis de las experiencias de las(os) profesionales de enfermería entrevistados se pudieron identificar herramientas adquiridas en el transcurso de su vida profesional, como también fortalezas y debilidades propias que presentan en la atención a una PVVIH. De esta manera se logró cumplir en su totalidad con el objetivo planteado, al conocer la percepción de enfermeras(os) respecto a la atención otorgada a PVVIH en un servicio hospitalario de atención terciaria de salud, logrando así contribuir a la disciplina de enfermería.

Sin embargo, luego de todo el proceso realizado y con la información obtenida se desprenden nuevas interrogantes en relación al tema expuesto, como: ¿en el pregrado se entregan herramientas necesarias para que las(os) futuros profesionales de enfermería enfrenten la atención de una PVVIH de manera integral?, ¿están siendo suficientes los esfuerzos del sector salud en Chile para detener la pandemia del VIH y fortalecer los cuidados que se otorgan a la población ya infectada?, o ¿el contexto sociocultural interfiere negativamente en el desarrollo de una atención holística e igualitaria por parte del sector salud hacia las PVVIH? Por otra parte, ¿cómo perciben las PVVIH la atención de las(os) profesionales de enfermería? o ¿qué esperan las PVVIH de la atención en salud y de las(os) profesionales de enfermería? De esta manera, se invita a seguir investigando con respecto a la atención que se otorga a esta población, para concientizar al profesional de enfermería con respecto a la atención entregada día a día en los diferentes contextos a los que se ve enfrentada(o).

Es por todo lo anteriormente mencionado que se hace tan necesario seguir estudiando y analizando la percepción que las(os) profesionales poseen en relación a la atención que se le brinda a la PVVIH, para así ir mejorando la relación terapéutica que se establece entre enfermera/o - paciente, reforzando fortalezas y herramientas identificadas e ir trabajando en aspectos a mejorar lo cual contribuirá al óptimo desempeño de la profesión, ya que contemplando la visión de las y los gestores del cuidado se podrá trabajar en mejoras continuas para suplir las falencias y reforzar lo ya logrado. 
Finalmente, considerando el actual aumento en las cifras de prevalencia de VIH en Chile, se hace fundamental que las(os) profesionales de enfermería hagan frente a esta situación, mejorando y reforzando habilidades blandas y procedimentales, conocimientos y aspectos éticos entre otros temas, para así optimizar el establecimiento de la relación terapéutica con la PVVIH y puedan contribuir en parte a la detención de este aumento, siendo agentes de cambio y educadores de toda la población.

Los datos obtenidos, si bien pueden ser aplicados para la mejora de la labor del profesional de enfermería, no son generalizables a todo el contexto existente actualmente, debido a la metodología del estudio; sin embargo se espera sean de utilidad y contribuyan en algún grado a la enfermería, permitiendo incentivar futuros estudios que aporten a la información obtenida y se puedan complementar o refutar los resultados expuestos en esta investigación, además de promover el rol investigador en las(os) profesionales de enfermería, explorando así nuevas áreas de desempeño disciplinar.

\section{REFERENCIAS BIBLIOGRÁFICAS}

1. 1. ONUSIDA. "El SIDA en cifras 2015" [en línea]. 2015 [citado el 11 de abril de 2017]. Disponible en: http://www.bibliotecaminsal.cl/wp/wpcontent/uploads/2016/02/Estadisticas-SIDA-AIDS_by_the_numbers_2015_es.pdf

2. Instituto de Salud Pública (ISP). Resultados confirmación de infección por VIH en Chile, 2010 - 2015. Boletín. 2016; 6(11): 3-12. [citado el 6 de abril de 2017]. Disponible en: http://www.ispch.cl/sites/default/files/BoletinVIH-15112017A.pdf

3. Vidal R, Adamuz J, Feliu P. Relación terapéutica: el pilar de la profesión enfermera. Enferm. glob. no.17 (Murcia). 2009. [citado el 9 de abril de 2017]. Disponible en: http://scielo.isciii.es/scielo.php?script=sci_arttext\&pid=S1695-61412009000300021

4. Registered Nurses Association of Ontario. Guías de buenas prácticas en enfermería: Establecimiento de la relación terapéutica. España; 2002. [citado el 8 de abril de 2017] Disponible en: http://rnao.ca/sites/rnaoca/files/2014_RTerapeutica_spp_022014_-_with_supplement.pdf

5. Duran M. El estudio de caso en la investigación cualitativa. Rev. nac. Adm; Volumen 3 (1):121-134.

6. Martínez-Salgado C. El muestreo en investigación cualitativa. Principios básicos y algunas controversias. Ciênc. saúde coletiva. [en línea] 2012; [citado el 8 de mayo de 2017]; 17 (3) Disponible en: http://www.scielo.br/pdf/csc/v17n3/v17n3a06.pdf

7. Emanuel E. ¿Qué hace que la investigación clínica sea ética? Siete requisitos éticos. En: Lolas F. Quezada A. Pautas éticas de investigación en sujetos humanos: nuevas perspectivas. OMS/OPS. Santiago; 2003. p. 83-95.

8. Jiménez E, Soledad M. Rigor científico en las prácticas de investigación cualitativa. Cienc. Docencia tecnol. [en línea] 2011; [citado el 4 de mayo de 2017]; 107-136. Disponible en: http://www.scielo.org.ar/scielo.php?script=sci_arttext\&pid=S185117162011000100004

9. Díaz-Bravo L, Torruco-García U, Martínez-Hernández M, Varela-Ruiz M. La entrevista, recurso flexible y dinámico. Investigación educ. médica. [en línea] 2013; 
[citado el 4 de mayo de 2017]; 2 (7): 162- 167. Disponible en: http://www.scielo.org.mx/scielo.php?script=sci_arttext\&pid=S200750572013000300009

10. Sayago S. El análisis del discurso como técnica de investigación cualitativa y cuantitativa en las ciencias sociales. Cinta Moebio [en línea] 2014 [citado el 12 de mayo de 2017]. Disponible en: http://www.facso.uchile.cl/publicaciones/moebio/49/sayago.html

11. Informe Nacional: evolución de la infección por VIH/SIDA Chile 1984-2012. Rev. chil. infectol. [En línea]. 2015 Feb; 32( Suppl 1 ): 17-43. [citado el 11 de diciembre de 2017]. Disponible en: https://www.google.com/url?q=http://www.scielo.cl/scielo.php?script\%3Dsci_artte $\mathrm{xt} \% 26 \mathrm{pid} \% 3 \mathrm{DS} 0716$ 10182015000100003\&sa=D\&ust=1513034497652000\&usg=AFQjCNHZPdWgJPt35AhAy3wzbAYijRLyg

12. Uribe Ana Fernanda, Orcasita Linda Teresa. Evaluación de conocimientos, actitudes, susceptibilidad y autoeficacia frente al VIH/sida en profesionales de la salud. av.enferm. [en línea]. 2011 [Citado el 11 de diciembre de 2017]; 29: 271-284. Disponible

en: https://revistas.unal.edu.co/index.php/avenferm/article/view/35821/37088

13. Espinosa Aguilar Anibal, Gibert Lamadrid María del Pilar, Oria Saavedra Michel. El desempeño de los profesionales de Enfermería. Rev Cubana Enfermer [en línea]. 2016 Mar [citado el 11 de diciembre 2017]; 32: 87-97. Disponible en: https://www.google.com/url?q=http://scielo.sld.cu/scielo.php?script\%3Dsci_arttext \%26pid\%3DS086403192016000100011\&sa=D\&ust=1513036282038000\&usg=AFQjCNEKbnjBpdQr KxVH9G7Ib7hUdeID2A

14. Madrid Gutiérrez Juan. Aspectos psicológicos y relacionales del paciente con ITS/vih. Una mirada más profunda a los aspectos a tener en cuenta en este tipo de consultas. Rev Española de Bioética (EIDON) [En linea].2016 Dic [citado el 11 de diciembre 2017]; 46: 24-33. Disponible en: http://www.revistaeidon.es/index.php/ficha/148/Aspectos-psicolgicos-yrelacionales-del-paciente-con-ITSvih-Una-mirada-ms-profunda-a-los-aspectos-atener-en-cuenta-en-este-tipo-de-consultas

15. Estefo Agüero Silvana, Paravic Klijn Tatiana. ENFERMERÍA EN EL ROL DE GESTORA DE LOS CUIDADOS. Cienc. enferm. [en línea]. 2010 Dic [citado el 11 de diciembre 2017]; 16: 33-39. Disponible en: http://www.scielo.cl/scielo.php?script=sci_arttext\&pid=S071795532010000300005

16. Ceballos, P. Desde los ámbitos de enfermería, analizando el cuidado humanizado. Revista Ciencia y Enfermería [en línea]. 2010 [citado el 16 de junio de 2017]; Vol. XVI, $\quad \mathrm{N}^{\circ} \quad 1 . \quad$ Disponible en: http://www.scielo.cl/scielo.php?script=sci_arttext\&pid=S071795532010000100004 